Archives

$20 \mid 1998$

Miroirs de la Raison d'Etat

\title{
La tactique du lierre : sur « l'état au miroir de la raison d'état ", de Marcel Gauchet
}

\section{Christian Jouhaud}

\section{(2) OpenEdition}

Journals

\section{Édition électronique}

URL : http://journals.openedition.org/ccrh/2534

DOI : $10.4000 /$ ccrh. 2534

ISSN : $1760-7906$

Éditeur

Centre de recherches historiques - EHESS

Édition imprimée

Date de publication : 12 avril 1998

ISSN : 0990-9141

Référence électronique

Christian Jouhaud, «La tactique du lierre : sur « l'état au miroir de la raison d'état », de Marcel Gauchet », Les Cahiers du Centre de Recherches Historiques [En ligne], 20 | 1998, mis en ligne le 20 avril 2009, consulté le 10 décembre 2020. URL : http://journals.openedition.org/ccrh/2534 ; DOI : https:// doi.org/10.4000/ccrh.2534

Ce document a été généré automatiquement le 10 décembre 2020.

Article L.111-1 du Code de la propriété intellectuelle. 


\title{
La tactique du lierre : sur « l'état au miroir de la raison d'état ", de Marcel Gauchet
}

\author{
Christian Jouhaud
}

1 «L'État au miroir de la raison d'État». L'article de Marcel Gauchet m'apparaît surtout comme une tentative magnifiquement réussie de lier la problématique du théologicopolitique dans la France moderne et celle de la formation d'un espace public critique, suscité, dans un apparent paradoxe, par la dynamique absolutiste. Précieux travail qui entraîne à penser avec lui. La dimension monumentale (par sa cohérence) de l'édifice théorique incite l'historien du politique non pas tant à le discuter qu'à l'emprunter, à y loger ses faits et ses hypothèses, à y grimper comme un lierre parasite, à l'occuper ainsi dans l'illégitimité d'une appropriation partielle et en partie arbitraire.

2 C'est ainsi que je voudrais d'abord y accrocher mon propos à partir de deux citations de Richelieu (page 220).

Première citation :

[...] les États n'ont pas de subsistance après ce monde, leur salut est présent ou nul. Seconde citation :

La raison et l'expérience nous montrent qu'il faut pour le bien de l'Église qu'il y ait balance entre les princes temporels, en sorte que dans leur égalité l'Église puisse subsister et se maintenir en ses fonctions et en sa splendeur [...].

C'est cette captation de religion par l'État dans le temps même de la dissociation des deux ordres [spirituel et temporel] qui constitue le point crucial [commente Marcel Gauchet]. Entendons le point crucial du processus de maturation de « l'État de la raison d'État ». La seconde citation propose un retournement assez spectaculaire et peut-être pas complètement dénué d'ironie, puisqu'il ne s'agit de rien moins que de regarder la chrétienté et son chef du point de vue du compromis politique mis en place en France par le régime de l'Edit de Nantes et le développement de ce qu'on nomme par commodité l'absolutisme (bourbonien). La monarchie française, qui a su récupérer et adapter le modèle de souveraineté mis au point par la monarchie spirituelle de la papauté, lui renvoie, comme par un miroir déformant, l'image d'une domination fondée sur 
l'arbitrage entre forces opposées qui fut en France la sortie politique de la crise des guerres de religion (une sortie par la «laïcisation» de l'État, modèle évidemment paradoxal pour la monarchie pontificale).

3 Le salut de l'État est présent ou nul : la sûreté, la conservation de l'État, l'accomplissement de sa puissance au service du public relèvent d'une sphère temporelle et y renvoient. Mais demeure une vision théologique des fins dernières de la politique; le salut purement temporel de l'État doit permettre d'assurer un ordre propice à la recherche du salut par les sujets qui vivent dans sa domination. L'État ne peut plus prétendre à la puissance d'instaurer la cité de Dieu sur terre qui garantirait le salut de la communauté politique, mais offrir les conditions propices à la préparation du salut des âmes. Les désordres, les troubles civils qui prospèrent quand l'État ne peut assurer ni manifester son salut temporel sont autant d'occasions de perdition pour ceux qui, refusant la dimension purement temporelle du salut de l'État (au nom même du salut spirituel de la communauté politique), participeraient à la ruine ou à la déstabilisation de l'État comme force purement temporelle.

4 Il y a donc un salut collectif temporel qui prépare le salut individuel des âmes. Mais ce salut collectif temporel, qui est le salut présent de l'État - conçu comme une charité due par le roi à ses sujets - s'identifie-t-il à une politique unique, celle finalement pratiquée par Richelieu, ou bien pourrait-il être porté par des politiques différentes? Dans le petit livre que nous avons publié sur La France du premier XVII siècle, nous avons, avec Robert Descimon, beaucoup insisté sur l'opposition de deux notions, celle d'ordinaire et celle d' extraordinaire qui renvoient à un fondamental choix opéré entre deux politiques qui, toutes deux, postulaient et visaient une expansion de la raison étatique. La première renvoyait à une vision coutumière de l'exercice du pouvoir, où les différentes institutions politiques de l'État exerceraient et développeraient leur rôle administratif et leur devoir de conseil du monarque. Elle n'excluait nullement une rationalisation administrative de la puissance étatique, l'expansion de ses prérogatives, le renforcement et l'exaltation de son autorité. La seconde installait le développement de la puissance étatique sur la connaissance confisquée, l'invention et le contrôle d'expédients, surtout financiers, permettant de conduire une politique de guerre. La première supposait le développement de la «bureaucratie rationnelle » des officiers, la seconde la puissance du favori et des clientèles qu'il était capable de mobiliser et de contrôler.

La victoire du système de l'extraordinaire avec Richelieu a eu plusieurs conséquences.

6 En premier lieu une capture, une confiscation si l'on veut, un détournement du processus de transfert de sacralité de l'Église sur l'État. Ce qui est dû, en grande partie, à la position spécifique de Richelieu et au dispositif de pouvoir qu'elle lui a permis de mettre en place. Comme chacun sait, Richelieu est un cardinal de l'Église romaine, prêtre, évêque, théologien, proviseur de la Sorbonne et abbé de plusieurs abbayes. Ce statut l'autorise à interpréter et à capter avec plus de légitimité et d'efficacité qu'un autre le lien maintenu, bien que réinterprété, de l'absolu du pouvoir d'État avec l'Absolu, et cela au moment même où la réinterprétation est produite et mise en actes. Prêtre, Richelieu prend soin de demander au pape les dispenses nécessaires pour l'exercice quotidien de sa fonction de premier ministre. Il s'emploie, d'autre part, à contrôler de fort près l'expression publique du spirituel, du miraculeux. Il surveille les débats théologiques. L'affaire Santarelli, la possession de Loudun, l'emprisonnement de l'abbé de Saint-Cyran en sont autant de manifestations. L'association avec le fameux Père Joseph, diplomate retors et sans scrupules et, en même temps, chef d'un ordre religieux strictement tenu en main, où les 
signes d'un lien maintenu au divin sont appelés, interprétés, exaltés, est un élément important du dispositif de pouvoir. C'est ainsi que le proprement religieux est réinstallé au cœur du dispositif de pouvoir, dans le cadre même d'une politique qui, par ailleurs, achève de rompre avec l'idéal de chrétienté. On pourrait dire que se trouve ainsi resacralisée, du côté du religieux, une sacralité déplacée vers l'État ou médiatisée par lui (j'utilise ici par commodité le terme de sacralité, sans prendre position sur ce qu'il recouvre, du point de vue anthropologique plus que religieux ; c'est évidemment tout un débat).

7 Cette entreprise soutient le dispositif de pouvoir mis en place par le cardinal, non seulement en ce qu'elle permet de produire comme croyance, mais aussi parce qu'elle définit la place centrale du favori (impose la centralité de cette place) à travers une position assumée tant à l'égard du roi que des agents de l'État. Sans définition institutionnelle claire, le pouvoir du ministre-cardinal sert de médiateur entre le roi et les agents de l'État en proposant une interprétation efficace des rapports entre l'Absolu divin et le pouvoir absolu du roi et donc de l'État. Il protège ceux qui détiennent des pouvoirs délégués de la hantise du péché dans l'exercice de leurs fonctions au service de l'État. C'est en cela aussi qu'il assure le salut présent de l'État, en libérant sa puissance d'action de la menace du péché. Pour le roi, il assume le péché de la violence d'État suscitée par le choix de la politique de l'extraordinaire (par principe la position du roi ne saurait être contaminée par l'extraordinaire : on le voit bien dans l'appel incessant qui est fait à lui contre celui-ci). Aux agents de l'État, il offre un pacte ; ils renoncent à percer les raisons cachées des décisions qu'ils ont à appliquer et ils se trouvent libérés du poids de toute culpabilité à l'égard de leurs actions d'agents de l'État. Ce pacte repose sur la postulation que le favori possède un double secret. Le secret des fins de la politique de l'État (et donc des moyens appropriés), ce qu'on peut nommer le secret des affaires, et aussi le secret de la nature du lien conservé avec l'Absolu désormais opaque. Dans le système de pouvoir mis en place par Richelieu, l'un ne va pas sans l'autre. Ce double secret d'État - dont la duplicité est fonctionnelle - n'a évidemment de virulence que parce que les politiques de l'État sont été désorbitées, par choix politique, des raisons prévisibles, coutumières et politiquement rationnelles de la gestion ordinaire des affaires. La postulation qu'il existe bien un lieu du secret d'État, dont il faut accepter l'éminente impénétrabilité, n'a vraiment d'importance que parce que s'imposent l'évidence des effets de la diplomatie occulte, l'épreuve des pratiques financières arbitraires, la liquidation des oppositions par l'intermédiaire de juridictions d'exception.

8 Cette pratique politique de la sacralité captée, fixée en un lieu secret, cultivée, retenue (comme on parle de justice retenue) a peut-être pour conséquence de détourner pour un temps le transfert de sacralité vers l'État (ce qu'on nomme la laïcisation de la politique) et donc de retarder son accomplissement final. En outre, cette captation ne peut se faire sans une théologie qui la favorise et l'accompagne. C'est tout l'enjeu de quelques débats qui traversent la première moitié $d u$ XVII ${ }^{\mathrm{e}}$ siècle, comme le débat sur la pénitence. On sait que Richelieu était attritionniste alors que ses opposants dévots étaient contritionnistes (le débat entre attritionnisme et contritionnisme n'avait pas été tranché par le concile de Trente). Si les agents de l'État en venaient à penser qu'ils ne pourraient être sauvés que par un mouvement positif de contrition et pas par le seul souci d'éviter la damnation, alors leur action publique serait inévitablement soumise à examen de conscience. C'est tout l'enjeu politique du premier jansénisme qui favorisait à la fois la désertion du service 
de l'État et le développement d'une self-consciousness chez ceux qui avaient vocation à le servir, association mortelle pour la bonne application des politiques de l'extraordinaire.

On peut donc dire que si le théologico-politique a bien été tenu à distance, avec succès, par la monarchie française, depuis la fin de la Ligue (avec le soutien actif des jurisconsultes gallicans), la théologie (tout court) fait pourtant retour comme enjeu sur le terrain politique par la mise en place des pratiques de l'extraordinaire et le renforcement de la raison d'État telle que l'imposent les cardinaux-ministres et leur dispositif de pouvoir. Avec le règne personnel de Louis XIV, le recentrement de l'extraordinaire sur la personne du souverain permettra de trancher dans le vif de ce retour politique de la théologie (liquidations de la Compagnie du Saint Sacrement, du jansénisme et du protestantisme, désignation du mouvement prophétique des camisards comme "fanatique »), mais ce sera au prix de l'absorption de la fonction royale dans la raison d'État de l'extraordinaire avec des conséquences à long terme mortelles pour le régime. Je voudrais donc émettre ici l'hypothèse que le processus de «désacralisation » de l'État n'est pas univoque et régulier. Il passe peut-être par cette phase de resacralisation que j'ai tenté de décrire trop vite, associée au développement d'une raison d'État de l'extraordinaire qui rompt le processus de rationalisation des pratiques étatiques amorcé au siècle précédent. C'est peut-être la question de l'impossibilité de l'évolution de la monarchie française vers une monarchique constitutionnelle qui surgit à l'horizon de cette question. En tout cas, l'État de la raison d'État s'est trouvé, dans son expansion, confronté au problème du choix entre des politiques différentes correspondant à des intérêts divergents. Il n'était pas écrit par avance que le chemin de l'extraordinaire serait la seule voie possible de la modernisation de l'État en France. C'est son choix qui a infléchi les pratiques de gouvernement non pas vers l'absolutisme à proprement parler (en un sens le cardinal de Bérulle, le garde des Sceaux Marillac, le duc d'Orléans et même le parlement de Paris étaient aussi absolutistes que Richelieu), mais vers une culture politique du secret, de l'expédient, concomitante d'un moment de brouillage intense des rapports entre l'Absolu de l'au-delà et l'absolu du pouvoir étatique dont «le salut est présent au nul».

10 La question de l'intérêt, ou plutôt des intérêts divergents, peut également faire retour - fantôme bourru et parasite - dans la dialectique du secret et de la publicité si brillamment décrite par Marcel Gauchet. Dans la partie de son article intitulée « Du secret à la publicité de la politique ", il défend l'idée, à première vue paradoxale, que l'État de la raison d'État rend " principiellement possible » la publicité de la politique.

Il évoque (p. 235), le pouvoir qui,

[...] par un côté, dégage ses maîtres de la contrainte commune et les soustrait à l'appréciation ordinaire au nom des suprêmes intérêts qu'ils ont à servir, par l'autre côté, rend leur conduite déchiffrable de l'extérieur, hors de toute connivence avec le monde de ceux qui sont dans le secret, sur la seule base de la pesée des situations. Ce qui fonde l'exception mystérieuse des voies de l'État est simultanément ce qui les expose à la sagacité de tout un chacun. Dans la mesure en effet où c'est l'inexorable loi de l'intérêt qui les guide et qui les justifie, elles sont susceptibles d'une objectivation qui autorise n'importe qui, idéalement, à en reconstituer du dehors l'enchaînement intelligible.

C'est ainsi que, virtuellement, se construit la place d'un

[...] point de vue du public [instauré par l'État] imposant le point de vue des intérêts qui s'imposent à lui. 
En outre, en exhibant la nécessité du secret, l'État installe dans le public la présence évidente d'un lieu mystérieux, dont l'entrée est interdite, mais dont on ne peut éviter de contempler le site. Le secret devient ainsi pour tous un objet de spéculation.

11 Si l'on passe de ce repérage d'une virtualité - fondamentale pour une histoire longue de l'espace public - au court terme des actions et de leur interprétation, se pose alors la question de l'identification du "quiconque», "n'importe qui », «tout un chacun» virtuellement dans la position de «reconstituer du dehors» les voies de l'État. Marcel Gauchet fait un pas dans cette direction quand il mentionne :

Sans doute l'information la plus étendue et la plus sûre se trouvera-t-elle souvent du côté des détenteurs de l'autorité. Mais il se pourra aussi qu'une situation soit mieux jugée de loin par un observateur impartial.

Si l'on considère ces deux figures « détenteurs de l'autorité » et « observateur impartial » comme des figures réelles, dans l'histoire, par exemple de la France du premier XVII siècle, on peut se demander s'il se trouve des acteurs réels pour les habiter. Où passerait la frontière entre les "détenteurs de l'autorité » et le public? Tout acteur politique, même rencontré pour sa seule opposition aux politiques de l'État, n'occupe-t-il pas conjointement les deux positions, soit personnellement, soit par l'intermédiaire du groupe (institution, clientèle, corps) par lequel il accède à l'action? Par ailleurs, la position d'observateur impartial, qui juge de loin, existait-elle, était-elle même pensable, voire imaginable? Cette position virtuelle, résultante d'un processus, peut-elle être occupée au XVII ${ }^{\mathrm{e}}$ siècle, ou bien ne serait-elle que la place de l'analyste moderne? $\mathrm{Ne}$ suppose-t-elle pas que soit comblé le décalage entre la raison des actions telle qu'elle pourrait être reconstituée dans l'action (par un acteur donc) et l'interprétation a posteriori des résultats? Se poserait alors la question de qui se trouve dans la position d'interpréter a posteriori, donc celle de l'identification des interprètes comme très différents de l'observateur impartial ou du « quiconque prend la peine de se placer devant les données effectives de la situation ».

Peut-être n'existe-t-il pas au XVII siècle d'observateur des intérêts (principe d'objectivation) qui soit lui même extérieur aux intérêts qu'il s'agit de reconstituer du dehors? Dans ce cas, l'intérêt (au singulier) comme principe de rationalisation et d'objectivation de la politique - on pourrait dire aussi comme fiction efficace qui permettrait d'objectiver et de rationaliser les comportements du pouvoir politique et donc d'en faire un objet de spéculation-, l'intérêt donc ne serait qu'un simulacre incertain. Il ne serait qu'un trompe l'œil, dissimulant la contradiction et donc l'imprévisibilité - sinon l'irrationalité - des intérêts affrontés, dont l'intérêt commun (la survie du système) ne constituerait qu'un socle, dont la restitution ne dirait que fort peu de chose sur la logique des actions, les stratégies et les tactiques des acteurs. Le court terme des actions resterait opaque.

Or, bien davantage qu'aujourd'hui, le court terme des actions où se cristallisaient les différences politiques et les oppositions d'intérêt était habité par des enjeux spécifiques, divers, mais compatibles, dans leur diversité même, avec les grands principes sur lesquels reposait le système politique, lequel, de ce fait, ne pouvait s'en trouver remis en cause. C'est ainsi, par exemple, que les logiques clientélaires qui continuaient d'organiser l'engagement politique, pouvaient certes être intégrées comme principe général et stable dans l'analyse d'une action politique, mais n'en restaient pas moins insaisissables au raisonnement dans leur configuration propre (et d'ailleurs mouvante). Le postulat partagé de leur efficacité ne suffisait nullement pour restituer les contours précis qui en 
faisaient la force spécifique. La reconstitution de ces configurations nécessitait un savoir auquel ne pouvait accéder - même virtuellement - un observateur extérieur. Seul un acteur bien placé pouvait y prétendre. Il n'était pas absolument impossible que cet acteur pût se transformer en médiateur qui aurait divulgué les secrets de cette configuration, (c'est sans doute l'une des clés du succès des mémoires comme genre mondain), mais cela signifiait que l'action était alors achevée et que ses enjeux propres avaient disparu. Dans l'action, l'acteur informé ne pouvait, au contraire, pour préserver ses intérêts propres d'acteur, que tenter de brouiller les fonctionnements de ces intérêts en action, sous peine de rendre son savoir inopérant dans l'action. Et, eût-il pris ce risque, que la divulgation de ce savoir aurait été reçue, par tous ceux qui ne voulaient pas être dupes, comme une action politique qui ne révélait le principe d'intérêt que comme un simulacre destiné à protéger des intérêts qu'il n'aurait pas été vraisemblable de voir accéder à la publication.

Pendant la Fronde, en face de tant de pamphlets qui prétendent occuper la position de l'observateur extérieur et produire des principes stables d'interprétation des actions, le lecteur averti cherche l'artifice, cherche les raisons cachées de cette écriture, celles qui échapperaient à tout principe restituable d'objectivation de la politique (les raisons rationnellement restituables de l'extérieur sont, dans cette perspective, à écarter d'emblée comme proprement impertinentes). À tel point que les critères de lisibilité de la politique du temps, présents - par exemple sous forme de maximes - dans les pamphlets, sont reçus par une partie non négligeable de leur public comme la preuve de son illisibilité, fussent-ils, par ailleurs, d'un haut degré de vraisemblance. Ils n'ont ainsi d'efficacité dans l'action politique que comme contraire direct de ce qu'ils prétendent être, sauf sur la part la plus «manipulable » des lecteurs qui seront supposés répondre par leur action irréfléchie aux sollicitations contradictoires de principes trompeusement stables, et ne réaliseront donc que des actions incohérentes à partir de principes apparemment cohérents.

Il ne s'agit pas tant de mensonge : la postulation d'une présence universelle du mensonge conduit, comme le remarque Marcel Gauchet, au moins à dire la vérité sur la présence du mensonge. Il s'agit plutôt d'une radicale incertitude sur la frontière du vrai et du faux et sur leurs rapports. Il s'agit d'une foncière indécidabilité de ce qui est vrai et de ce qui est faux. Et de la conscience aiguë qu'il faut, pour en éviter le piège, être capable de mobiliser un point de vue d'acteur sur l'action et non d'observateur, qu'il faut être capable de mobiliser un savoir absent de tous les lieux où les principes d'objectivation de la politique pourraient être rendus publics.

Ce règne de l'équivoque dans la politique du temps attire l'attention sur ceux qui en sont les théoriciens et les praticiens. Louis Marin avait naguère insisté sur un échange fondamental entre les techniques du coup politique-associant secret et ostentation fulgurante des effets - et techniques rhétoriques de la persuasion, les premières servant de modèle à la mise en action des secondes dans des textes, les secondes offrant aux premières l'expérimentation de la performativité. Pareils échanges qui montrent que, par exemple, une théorie de l'action dramatique peut se substituer à une " science politique " défaillante pour fournir au pouvoir une théorie de l'action en général, invite à bien mesurer l'importance du processus qui voit l'éloquence publique et civique des assemblées, des corps politiques, perdre son efficacité et son influence politique au profit d'un simulacre délocalisé (hors des institutions politiques) qu'on peut nommer « littérature ». Ce processus de littérarisation de l'éloquence produit un bouleversement des critères qui permettent d'apprécier et de légitimer l'usage de celle-ci, mais il 
déstabilise aussi les rapports de la parole ou de l'écrit public à la vérité et au mensonge. Il brouille la relation entre ceux qui parlent ou écrivent et ceux qui reçoivent et s'approprient ces paroles ou ces textes, entre les acteurs de la politique et d'éventuels observateurs. Entre ceux-ci et ceux-là se tient désormais le groupe informe - hors statut et hors institution-des médiateurs qui, faute de "tribunal de l'opinion publique", mesurent leur efficacité et leur utilité à la virtuosité de pratiques appréciées et jugées dans leur propre milieu et par ceux qui les emploient (les grands acteurs politiques), pratiques dont l'équivoque est la reine.

17 Il me semble qu'il faut voir là une limite à la capacité de l'État de la raison d'État à produire virtuellement un espace public de la critique à partir de ses pratiques de dissimulation qui seraient lisibles et questionnables comme telles. Non que ce modèle d'interprétation de ce qu'on pourrait appeler l'énergie de la raison d'État n'ait pas de sens, bien au contraire. Mais le rôle des acteurs sociaux de la médiation entre les acteurs de la politique et leurs destinataires (et éventuels "observateurs ») doit être analysé comme une captation, une retenue de cette énergie. Et donc comme le chaînon indispensable pour comprendre la dialectique du secret et de la publicité dans l'État d'Ancien Régime. L'action propre de ces médiateurs et la relation qu'ils établissent avec un public qui n'est pas le public de la politique (relation qui permet leur montée en puissance) finissent par réorienter cette énergie captée vers une publicité qui, dans l'opération, aura changé de sens. Les techniciens de l'équivoque, dont les prouesses révélaient aussi l'extraordinaire équivocité du statut, deviendront finalement les porteparole du tribunal de l'opinion publique. Au départ, la position d'observateur impartial était déniée à tout destinataire réel du jeu des équivoques, et comme éradiquée de la communication politique entre gouvernants et gouvernés. À l'arrivée une autorité morale, que les médiateurs occupent par simulacre de délégation, substitue le règne de la critique à celui de la manipulation: d'un simulacre à l'autre, d'une efficacité à l'autre. Pour le dire autrement, dans les deux cas, la place de l'observateur extérieur est occupée par des médiateurs qui sont aussi à l'intérieur et défendent, à l'intérieur comme à l'extérieur, leurs intérêts propres; le simulacre d'extériorité du jugement (qu'ils savaient à la fois produire et vider de toute efficacité politique), qui leur permettait de servir le pouvoir encourageant leur montée en puissance, sera finalement occupée par euxmêmes, au nom d'une puissance morale dont ils s'emploieront à dessiner le visage multiforme, dotant ainsi le leurre d'une efficacité que leurs commanditaires n'avait pas su imaginer.

18 L'histoire est le premier genre littéraire où se trouve installée la figure du destinataire comme observateur et juge. Le tribunal de l'histoire qui révèle a posteriori la vérité sur les mensonges de l'action politique et aussi sur les passions qui l'habitent, qui révèle le dessous des cartes, peut probablement apparaitre comme le laboratoire où s'expérimente la conversion du manipulateur au service du pouvoir en juge du commanditaire qui l'entretient (ou l'a entretenu). Il faudrait étudier l'histoire de la fonction d'historiographe de ce point de vue-là. Et peut-être aussi poser une question que je trouve assez troublante : pourquoi la monarchie française et ses ministres, malgré des efforts qu'on peut qualifier de colossaux, malgré de nombreuses et originales tentatives à la performativité puissamment conçue, n'ont-ils pu obtenir de leurs serviteurs de plume au long du xvII siècle l'écriture d'une histoire contemporaine qui les satisfasse, dans laquelle ils auraient pu se reconnaître comme les grands acteurs rationnels de l'État de la raison d'État? 